\title{
CFD Simulation on Heat Exchanger Cooled Dry-type Transformers
}

\author{
Wei Wu' ${ }^{1}$, Yong Wang', Zepu Wang ${ }^{3}$ \\ ${ }^{1,3}$ ABB Inc. \\ 901 Main Campus Drive, Raleigh, USA \\ wei.wu@us.abb.com; zepu.wang@us.abb.com \\ ${ }^{2} \mathrm{ABB} A G$ \\ Keffelker Str. 66, Brilon, Germany \\ yong.x.wang@de.abb.com
}

\begin{abstract}
Dry-type transformer applications are growing in transformer market because the technology is non-flammable, safer and environmental friendly. Since no oil is present in a dry-type transformer for dielectric insulating or cooling purposes, the unit size is normally larger and as such material cost becomes higher. Therefore how to design a dry-type transformer with well-balanced dimension and performances becomes one of the primary tasks of a transformer manufacturer. In particular, water cooling heat exchangers used for marine or off-shore platform transformer applications can greatly enhance the cooling performance of the transformers. In order to optimize the dry-type transformer products with heat exchangers, the present paper introduces computational fluid dynamics ("CFD") simulation tool to evaluate the cooling designs. By comparing the simulation results with the experimental results obtained from testing, the CFD models show acceptable accuracies; thus the verified technology is employed for the optimization of the cooling designs of the transformer products.
\end{abstract}

Keywords: Dry-type transformer, heat exchanger, optimization, temperature rise, thermal model, CFD

\section{Introduction}

Electrical transformers convert electricity from one voltage to another voltage, either of lower or higher values; as such they are key components of electric transmission and distribution networks. Among various transformer technologies, dry-type technologies avoid using oil as dielectric and cooling media and, therefore, becomes a non-flammable, safer and more environmental friendly alternative to liquid-filled transformer technologies. Because of these advantages, dry-type transformers have gained market share in special environments and applications which require high standard of safety and reliability, such as urban areas, buildings, off-shore platforms and marine ships etc.

However, one of the disadvantages of dry-type transformers is that they normally require larger dimensions, (therefore increased material costs), to have sufficient cooling and to constrain the operation temperature of the transformers within a specific range, considering low temperatures will help guarantee low level of insulation degradation rate. IEEE and IEC standards both have winding temperature rise requirements for dry-type transformers and according to the standards products manufactured need to pass heat-run tests before they can be finally equipped for operation. In this context one of the primary tasks of product designers and engineers becomes to find a way to predict temperature rises during their design stage and make sure products have enough cooling performance to ensure successful tests.

Dry-type transformers are usually cooled by either air natural (AN) flow or air forced (AF) flow; a special family of dry-type transformers are cooled by external heat exchangers, for instance, using water as the coolant. Figure 1 shows a transformer example with water cooling heat exchangers. In environments where large amount of low temperature water can be utilized, such as marine or off-shore platform applications, water cooling heat exchangers can significantly improve the cooling performance of the transformers. In order to optimize the transformer products with heat exchangers, design tools to predict the temperature rises then become of great importance. 


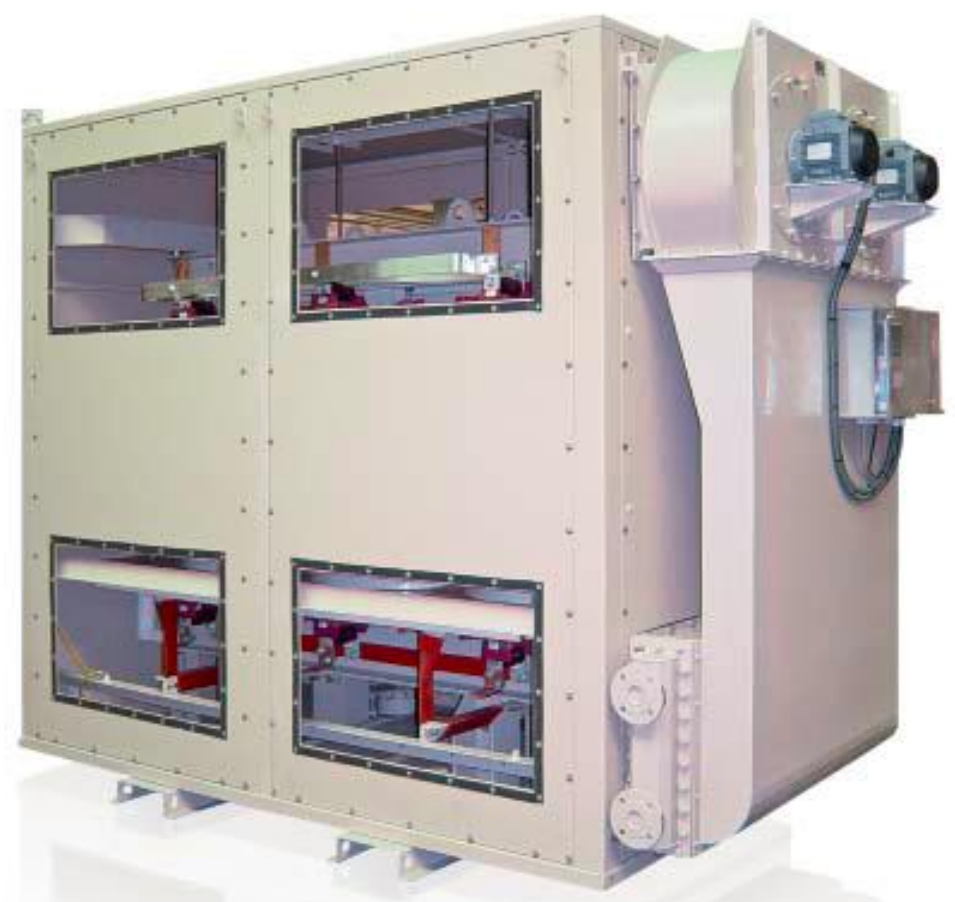

Fig. 1: Dry-type transformers with water cooling heat exchangers.

Along with the computer technology development, numerical modelling is gradually applied to predict the transformer winding temperature rises. The numerical approaches are generally classified into two categories; either computational fluid dynamics (CFD) [1,2] or numerical solutions which incorporate a degree of 'thermal network models' [3-7]. Thermal network models employ lumped parameter modelling philosophy which, based on the analogy with electric circuits, abstracts the phenomena of thermodynamics and fluid dynamics occurring in the computational domain into a number of interconnected so-called 'lumped elements' and the physics principles are encapsulated into the elements for numerical solutions. Compared to thermal network models, CFD is a numerical method with much higher level of discretization and, as such, will consume much more computational resources (CPU and memory) and time. Considering the benefit of CFD including its flexibility and that it can reveal more details of the thermal and flow phenomena, their application for transformer product design and development is increasing.

\section{CFD Modelling and Test Case}

Figure 2 (a) shows the geometry structure of a dry-type transformer with heat exchangers, which can be used as a test case. The heat exchanger is externally from the enclosure; it provides from the lower side of the enclosure cool air. The air turns direction, vertically flows through the three phase coils, picks up heat generated in the windings and the air's temperature then goes up. The warm air is sucked by the heat exchanger fans equipped from the upper side of the enclosure; in this manner the warm air flows back to the heat exchanger and dissipates its heat to the water circulation. In order to guarantee as much as possible air flowing through the heat generating coil sections, an air guiding plate is usually arranged to block the by-pass of the air. In manufacturing process there might be space between the air guiding plate and the outer high voltage windings; this space is defined as the air gap of the air guiding plate.

For numerical simulations with acceptable computational time, the geometry need to be simplified. First only a single phase coil is considered to be the average of the three phases, although in practice the middle phase may have worse cooling situations. Second the single phase coil is considered as cylindrical and the case can be collapsed into a 2-dimensional axial symmetrical model in order for computational load reduction. The reduced model is illustrated in Fig. 2 (b); which comprises the major components including the core, the low voltage and high voltage windings, the air guiding plate and the enclosure wall at the right-hand side. The left-hand side boundary is the symmetry axis. The inlet boundary at the domain bottom is set as a velocity boundary condition whose velocity value can be assessed from the heat exchanger's specification; the outlet boundary at the top is a pressure outlet boundary condition whose pressure can be set as zero to be the pressure reference. 


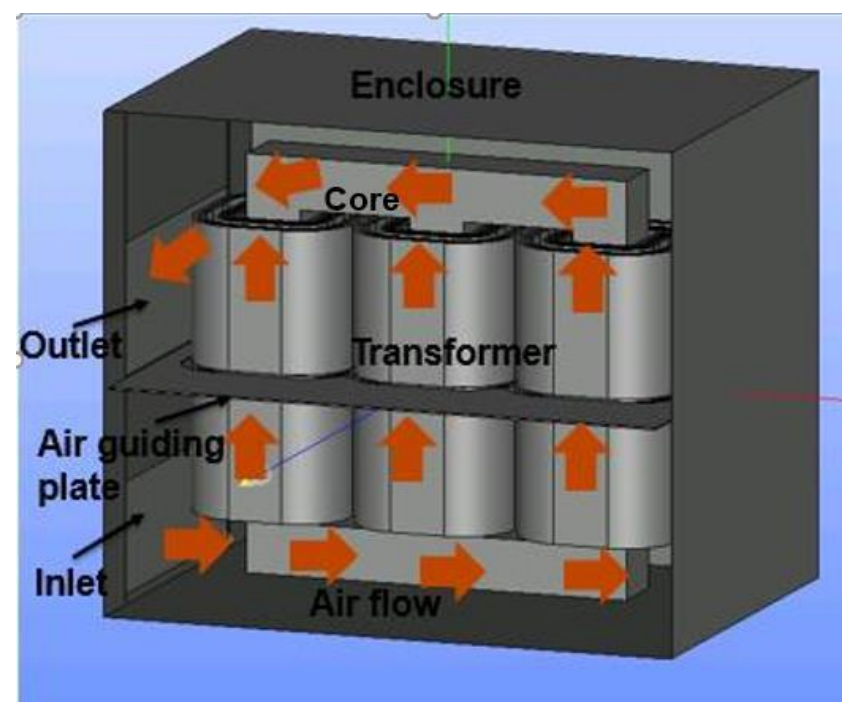

1

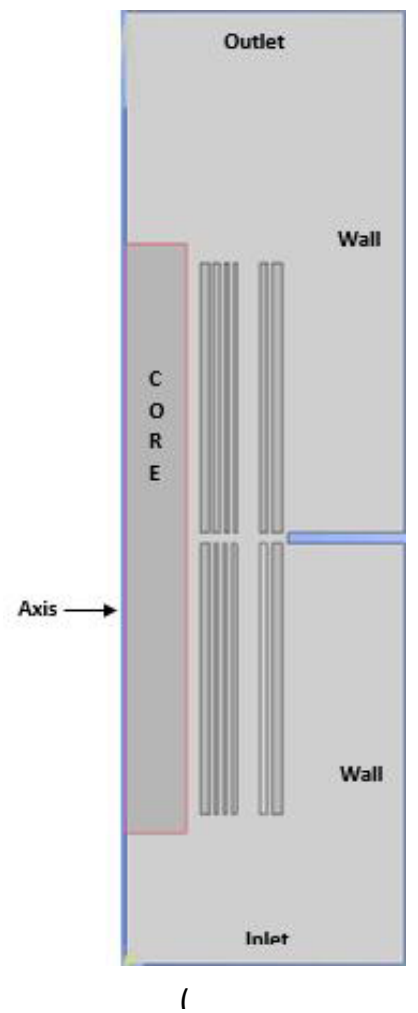

1

Fig. 2: Dry-type transformer test case with water cooling heat exchanger. (a) Transformer geometry structure including the three phase core, coil, air guiding plate and enclosure; (b) 2D extraction of the transformer geometry.

The 2D geometry model was built and meshed by using SALOME software; the CFD model was implemented using ANSYS Fluent 14.0. For the test case unit shown in Fig. 2, the CFD model is calculated and the temperature distribution is thus obtained and illustrated in Fig. 3. The accuracies of the numerical solutions were validated by referring the closeness of the CFD modelling solutions to the experimental test results. It is observed from Table 1 that the CFD results are in close agreement with the experimental results. The larger deviation (CFD - experimental results) of the low voltage winding II and the high voltage winding can be due to the presence of a screen between them which might affect the temperature rises of both. Secondly, in the 2D model the neglected cooling duct ribs can contribute towards the temperature rise deviations as well.

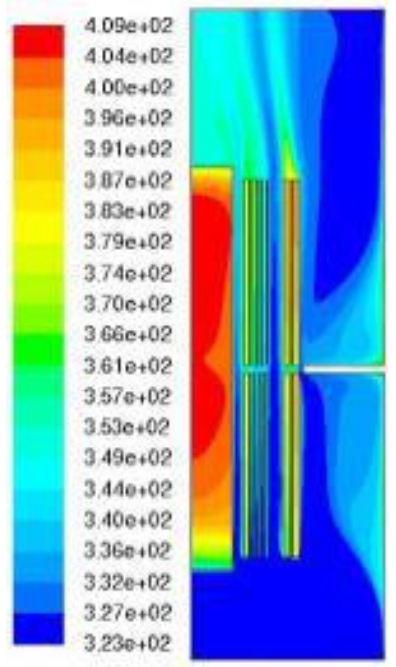

Fig. 3: Temperature (in K) distribution of the transformer test case. 
Table 1: CFD versus Experimental results.

\begin{tabular}{|c|c|c|c|}
\hline Component & $\begin{array}{l}\text { Temperature rise } \\
\text { (CFD), K }\end{array}$ & $\begin{array}{l}\text { Temperature rise } \\
\text { (Experimental), K }\end{array}$ & $\begin{array}{l}\text { Deviation (CFD - } \\
\text { Experimental) }\end{array}$ \\
\hline Core & 77 & 78 & -1 \\
\hline Low voltage winding I & 36 & 34 & 2 \\
\hline Low voltage winding II & 42 & 46 & -4 \\
\hline High voltage winding & 65 & 70 & -5 \\
\hline
\end{tabular}

\section{Parametric Study with CFD}

The validated CFD modelling tool can be used to study how different sizes of the air gap and different locations of the air guiding plate will affect the transformer's cooling performance as well as the winding temperature rises.

Figure 4 to 6 show the pressure, axial air flow velocity, temperature distributions respectively for the different sizes of the air guiding plate gap. As Fig. 4 shows, the pressure drop built by the air guiding plate will be dramatically reduced by the enlarged sizes of the plate gap. Lower pressure drop then reduces the velocity magnitude of the air flowing through each of the cooling duct inside the coils and results in high winding temperature rises; the distributions of the axial air velocity and temperature are illustrated in Fig. 5 and 6 respectively. Hence, the smaller air guiding plate gap contributes to lower temperature rises and as such is recommended.
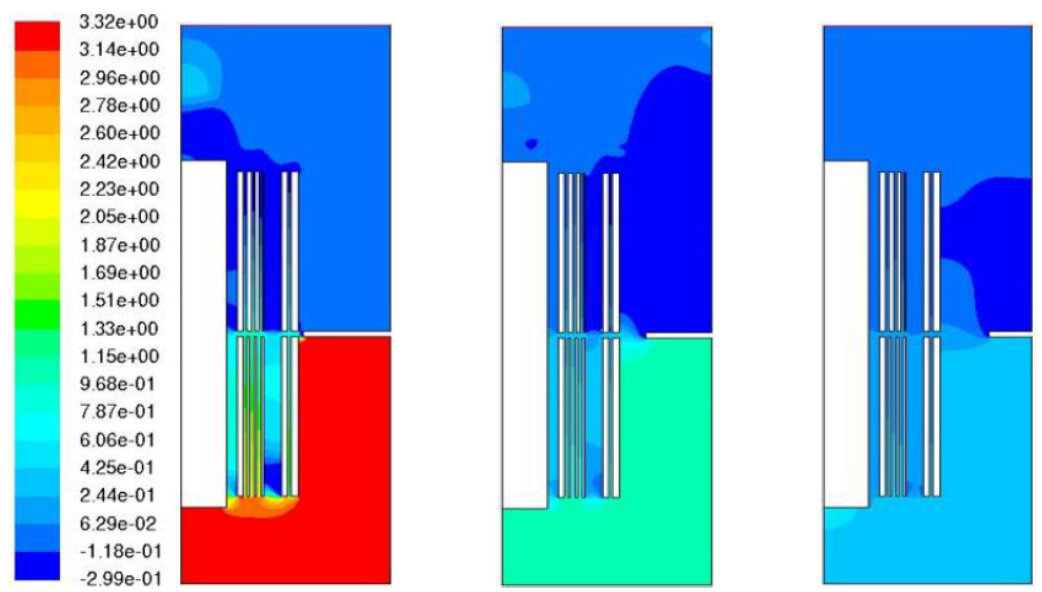

Fig. 4: Pressure (in Pa) distribution comparison for different sizes of the air guiding plate gap. (a) Standard size of air gap; (b) Enlarged size of air gap; (c) More enlarged size of air gap.
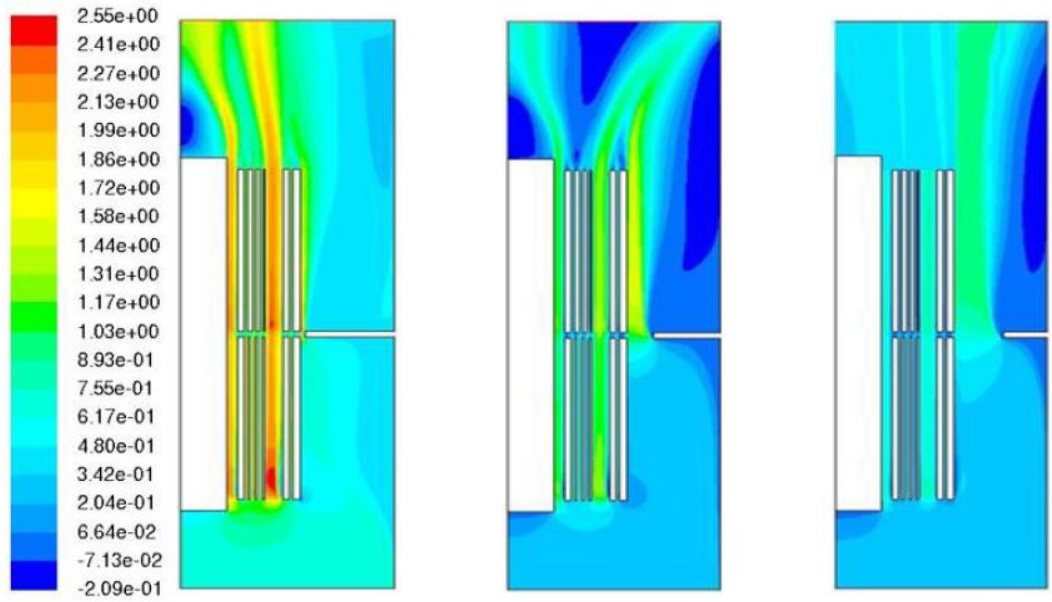

Fig. 5: Axial air velocity (in $\mathrm{m} / \mathrm{s}$ ) distribution comparison for different sizes of the air guiding plate gap. (a) Standard size of air gap; (b) Enlarged size of air gap; (c) More enlarged size of air gap. 

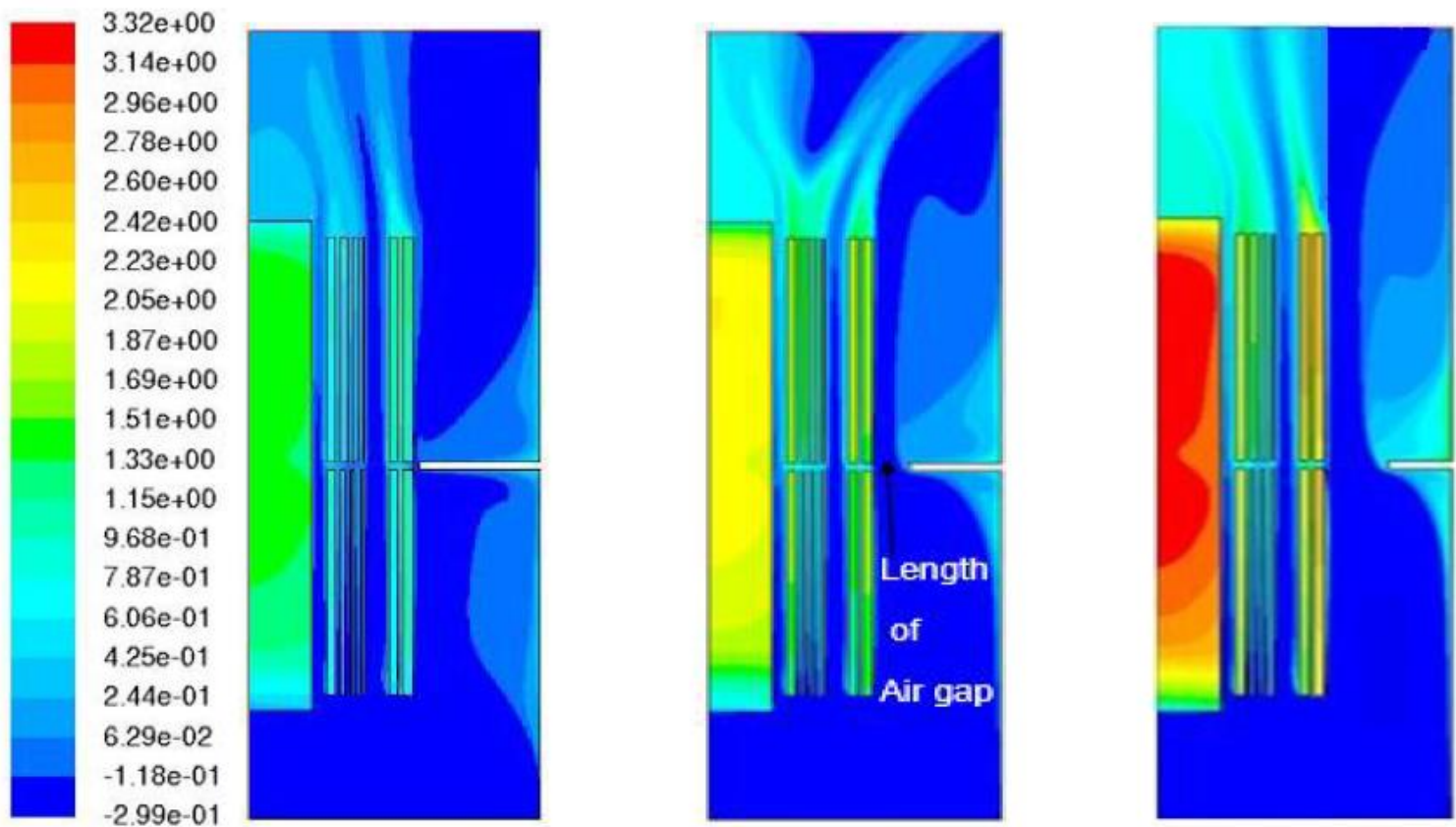

Fig. 6: Temperature (in K) distribution comparison for different sizes of the air guiding plate gap. (a) Standard size of air gap; (b) Enlarged size of air gap; (c) More enlarged size of air gap.

Figure 7 and 8 show the axial air velocity and temperature distributions for the different air guiding plate positions respectively. Fig. 7 shows that the different plate positions do not alter the air velocity magnitude inside the coils much; however the air velocity along the outer surface of the high voltage winding is affected to some extent. This is then the reason why in Fig. 8 the temperature rises of the core and the low voltage windings are comparable between the three plate positions; on the other hand, regarding the high voltage winding, the bottom plate position will make the upper part of the winding overheated, (so-called 'hot-spot'), the top position causes the lower part overheated, and the middle position configuration of the air guiding plate yields more homogeneous heating for the high voltage winding, which is therefore considered to be the optimal design when the winding temperature rises are concerned.
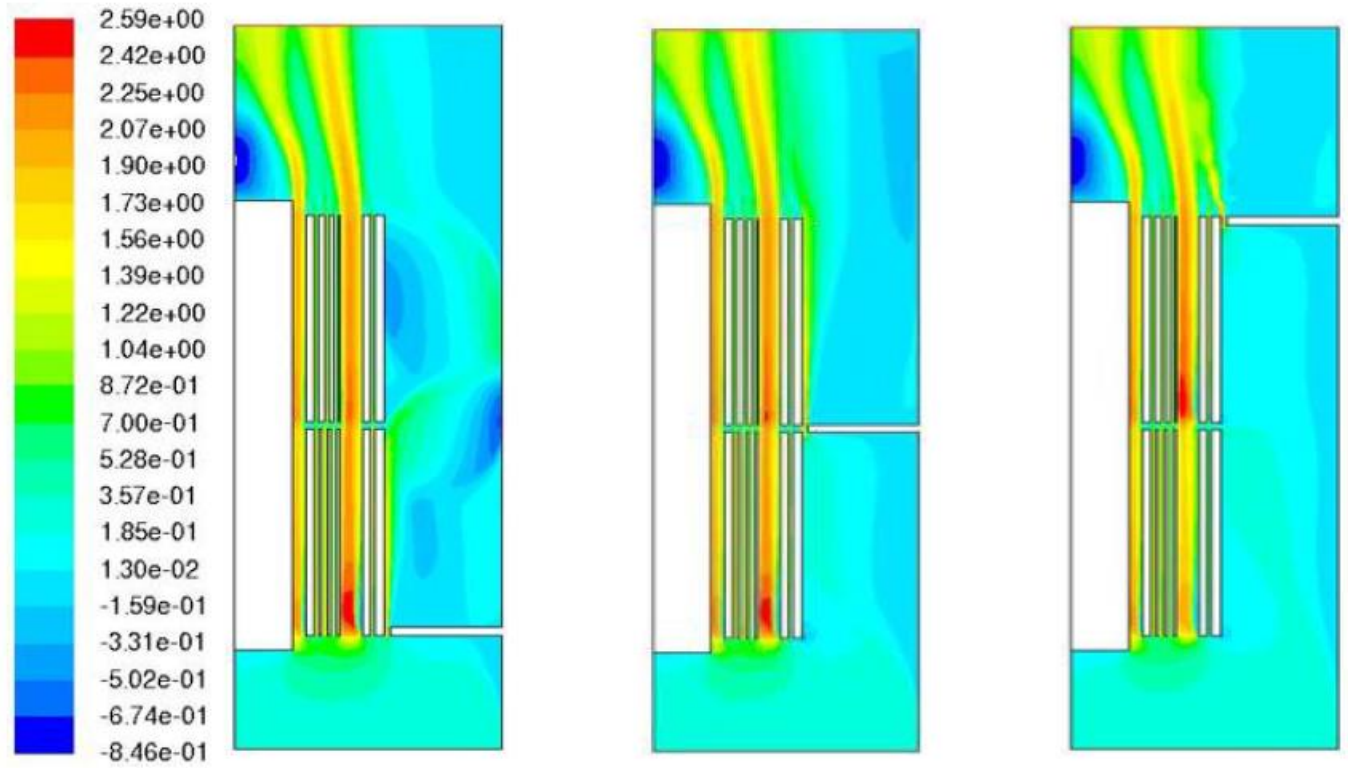

Fig. 7: Axial air velocity (in $\mathrm{m} / \mathrm{s}$ ) distribution comparison for different positions of the air guiding plate. (a) Bottom position; (b) Middle position (standard position); (c) Top position. 

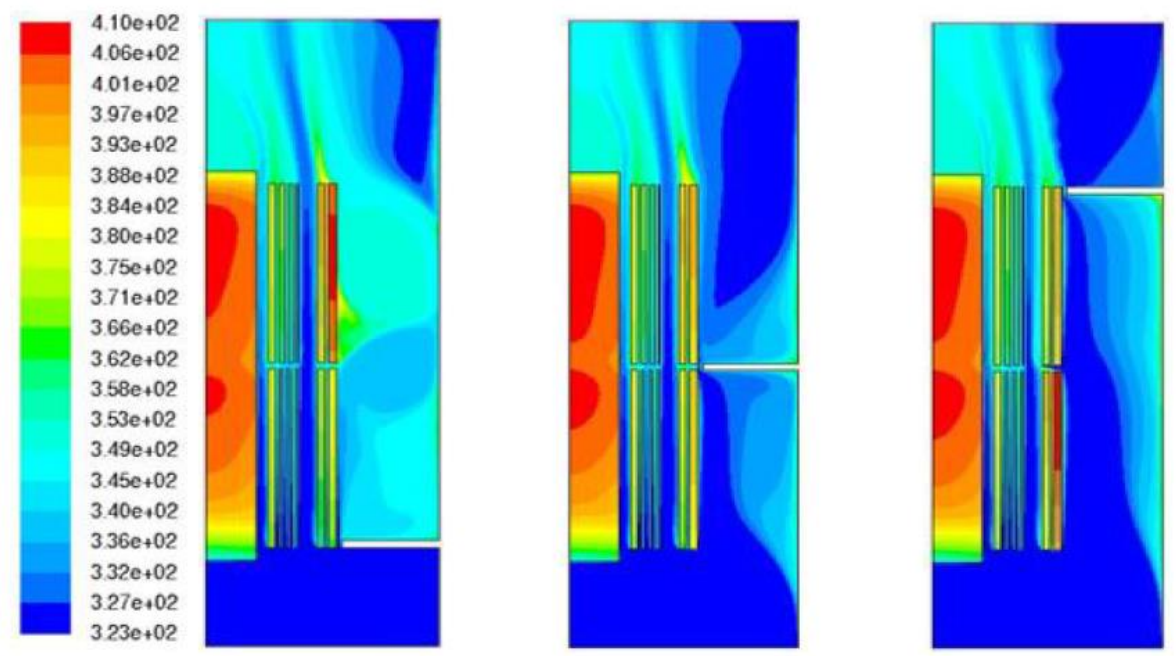

Fig. 8: Temperature (in K) distribution comparison for different positions of the air guiding plate. (a) Bottom position; (b) Middle position (standard position); (c) Top position.

\section{Conclusion}

The CFD model presented in this paper provides an accurate numerical approach to study the cooling effect of a drytype transformer unit with external heat exchangers; compared with laboratory experiments, the approach is also easier and takes lower cost. In the present paper, by taking two series of parametric studies as examples, it is demonstrated that CFD simulation tools have the great potential to be applied in practice either to derive design guidelines for transformer cooling or to assist the design and development of transformer products.

\section{Acknowledgements}

Due appreciation should be given to our MSc project student Mr Naveen Hariprasad who carried out the initial investigation of the idea in this paper under the supervision of the authors.

\section{References}

[1] A. Weinlaeder, W. Wu, S. Tenbohlen, and Z. Wang, "Prediction of the oil flow distribution in oil-immersed transformer windings by network modelling and computational fluid dynamics," IET Electr. Power Appl., vol. 6, no. 2, pp. 82-90, 2012.

[2] R. B. Fdhila, J. Kranenborg, and T. Laneryd, "C.-O. Olsson and B. Samuelsson, "Thermal modeling of power transformer radiators using a porous medium based CFD approach," THERMACOMP 2011, Dalian, China, 2011.

[3] W. Wu, Z. Wang, A. Revell, H. Iacovides, and P. Jarman, "Computational fluid dynamics calibration for network modelling of transformer cooling oil flows - Part I: heat transfer in oil ducts," IET Electr. Power Appl., vol. 6, no. 1, pp. 19-27, 2012.

[4] W. Wu, Z. Wang, A. Revell, and P. Jarman, "Computational fluid dynamics calibration for network modelling of transformer cooling oil flows - Part II: pressure loss at junction nodes," IET Electr. Power Appl., vol. 6, no. 1, pp. 2834, 2012.

[5] A. Blaszczyk, R. Flueckiger, T. Mueller, and C.-O. Olsson, "Convergence behavior of coupled pressure and thermal networks," Compel Journal, vol. 33, no. 4, pp. 1233-1250, 2014.

[6] E. Morelli, P. Di Barba, B. Cranganu-Cretu, and A. Blaszczyk, "Network based cooling models for dry transformers," ARWtr Conference, Baiona, Spain, 2013.

[7] A. Cremasco, P. D. Barba, B. Cranganu-Cretu, W. Wu and A. Blaszczyk, "Thermal simulations for optimization of dry transformers cooling system," The 10th Scientific Computing in Electrical Engineering (SCEE), Wuppertal, Germany, 2014. 